\title{
Mass Moment Determination Using Compound Pendulum
}

\author{
Zdzisław Pluta, Tadeusz Hryniewicz* \\ Koszalin University of Technology, Raclawicka 15-17, PL 75-620 Koszalin, Poland \\ *E-mail address: Tadeusz.Hryniewicz@tu.koszalin.pl
}

„People fall down due to the truth from time to time but most of them rise and walk off quickly allegedly nothing happened"

\author{
Winston Churchill
}

\begin{abstract}
This work has been performed to verify the existent knowledge on determination of the mass moment. For the experiment, a compound pendulum was used. The motivation to undertake these studies were experimental results indicating a big discrepancy in mass moments between the values coming from calculations using the definition formula and these obtained from the experiment. In relation to the axial moment the relative error equals $23.6 \%$, whereas regarding the polar moment the error reached $56.4 \%$. Considering the reason of that discrepancy we could find the existent theory not to be adequate. The theory is then considered in view of verifying first the mathematical pendulum and next the physical/ compound pendulum theory. The consideration has been focused on the description of accelerated motion cycle of both pendulums as it was enough to solve the problem. A source differential equation, which serves to solve any quantum phenomena, was used in the study. Then the course of creation of detailed characteristics of the phase of mathematical pendulum accelerated motion is presented as the basis to derive formula on the mass moment of a compound pendulum. At the end this new adequate theory was verified showing the relative error to be less than one per cent.
\end{abstract}

Keywords: Mathematical pendulum; Compound pendulum; Mass moment; Angular acceleration; Inertia moment; Time constant; Gravitation; Pendulum vibration period

\section{INTRODUCTION}

In his „Logic of scientific discovery” Popper [1] writes: „In the history of science, always theory, not experiment, idea and not observation, open the way to a new knowledge; I am also convinced that just the experiment saves us from making for nowhere; experiment allows getting out of old ruts and calls for finding new ways/paths." The essential meaning of experiment is also accented in [2], indicating the mind to cooperate with the matter and the scientist's intellect with techniques and technology. If the knowledge is inadequate then it has 
to be excluded and replaced by a new one adequate theory. Maybe the discrepancies between the theory and practice are noticed or even experienced but, for the time being, ignored to walk off quickly as if nothing happened [3].

Authors of the work used to penetrate the existent knowledge to find numerous and clear discrepancies from the reality. The cognitive actions and trials are undertaken to describe the studied phenomena in an adequate way [4]. This work, concerned with a new adequate description of mathematical pendulum and compound pendulum, is the example of this approach. It is worthy admitting that the thesis of inadequacy of the existent descriptions of these pendulums was formulated after an in-depth analysis of the variable motion of material bodies. The experimental results are delivered to confirm this thesis. Also a proper description of kinetics of the two pendulums is presented.

The idea to undertake this subject was born much earlier and now it was confirmed by the experimental results. This experiment was to determine the mass moment of a material body.

At the beginning, however, before the development of the thesis, let us clarify the notion of mass moment. It exists in science as the inertia mass moment or just inertia moment, without a necessary strictness. This last notion does not specify if it is concerned on surface or mass. Anyway, it is not about the inertia moment as its essence is quite different. It is worthy presenting analytical form of this physical magnitude, then

$$
M_{B}=J \cdot \varepsilon
$$

The inertia moment $M_{B}$ is equal to the product of the inertia moment $J$ and the angular acceleration $\varepsilon$ ? That does not make any sense! A cardinal terminological error has occurred; the rule of univocality [5]. That rule means the name to be referred to one notion; one cannot use ambiguous multivocal names meaning different notions (homonyms).

Therefore it is justified to differentiate the names of these magnitudes, i.e. $M_{B}$ and $J$. This first symbol determines then the inertia moment, whereas the second one - the mass moment. The inertia moment $M_{B}$ is equal to the product of the mass moment $J$ and the angular acceleration $\varepsilon$, and it is the right reading of the formula (1) on the inertia moment of a material body.

\section{EXPERIMENTAL}

The subjected material body is presented in Fig. 1, with the mass moment determined by an experiment. This is the ring of the measured mass $m$ equaling to $74 \mathrm{~g}$, that is $0.074 \mathrm{~kg}$.

The outer diameter of the ring equals $D=94 \mathrm{~mm}$, or $0.094 \mathrm{~m}$, with the inner diameter $d$ $=84 \mathrm{~mm}$, or $0.084 \mathrm{~m}$. 

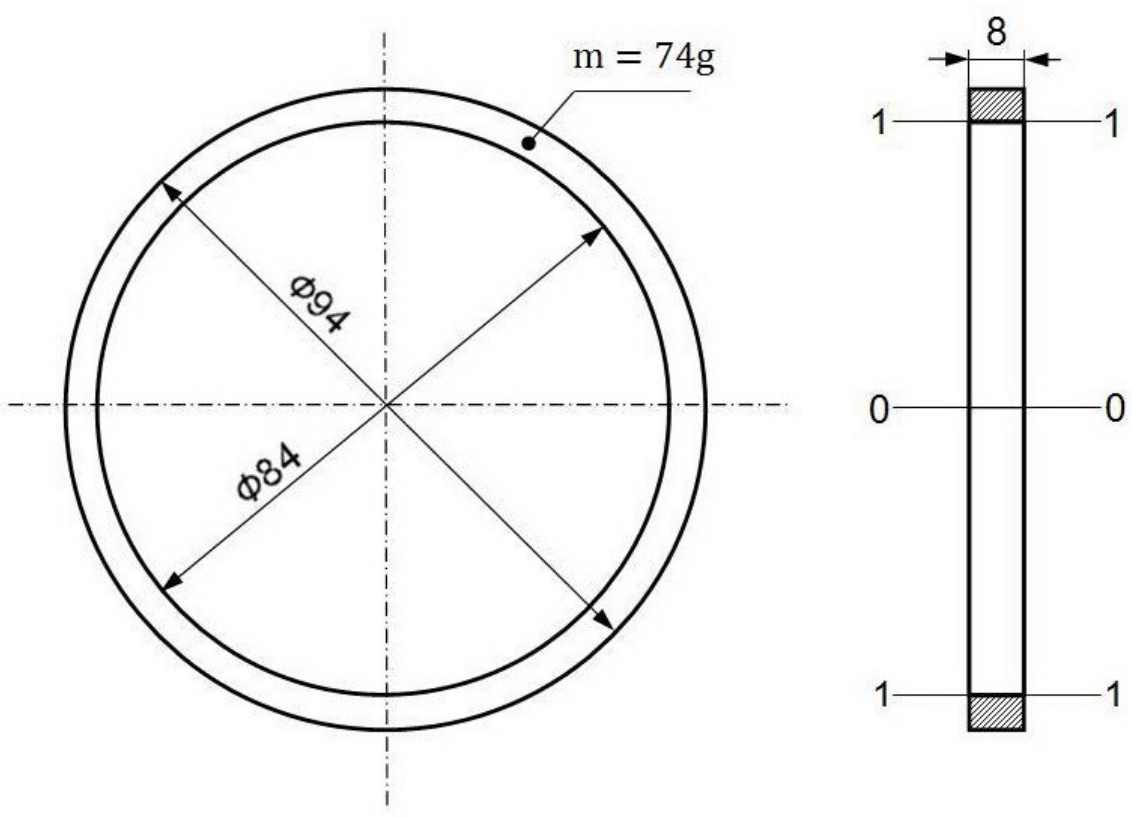

Fig. 1. Characteristics of the studied ring

A known formula on the polar mass moment $J_{0}$, that is the mass moment against the axis coming through the solid centre, takes the following form:

$$
J_{0}=\frac{m}{2}\left(R^{2}+r^{2}\right)
$$

where $R$ determines the outer radius, and $r$ - the inner radius of the ring, then $R=D: 2$; $r=d: 2$.

Substituting the results of measurements characterizing that ring, with the magnitudes $m=0.074 \mathrm{~kg} ; R=0.047 \mathrm{~m} ; r=0.042 \mathrm{~m}$, one obtains

$$
J_{0}=\frac{0.074}{2}\left[(0.047)^{2}+(0.042)^{2}\right]=0.147 \cdot 10^{-3} \mathrm{~kg} . \mathrm{m}^{2}
$$

And now let us derive the mass moment $J_{1}$, that is this kind of moment against the axis coming through the periphery of the ring's hole (see Fig. 1). That moment is determined by a Steiner theorem, namely

$$
J_{1}=J_{0}+m r^{2}
$$

where $r$ is the distance between the considered axes, i.e. $0-0$ and 1-1. That distance is equal to the inner radius of the ring. 
By substituting the values of the magnitudes: $J_{0}=0.147 \times 10^{-3} \mathrm{~kg} \cdot \mathrm{m}^{2} ; m=0.074 \mathrm{~kg} ; r=$ $0.42 \mathrm{~m}$, to the formula (3), one obtains

$$
J_{1}=0.147 \cdot 10^{-3}+0.074(0.042)^{2}=0.278 \cdot 10^{-3} \mathrm{~kg} \cdot \mathrm{m}^{2}
$$

Thus the process of determination of the moments $J_{0}$ and $J_{1}$ could be considered as completed. The moments have been determined and the aim achieved. However, in fact it is just the beginning of the titled problem: now it should be referred to the pendulums, mathematical and a compound one. In the further course of actions an essential cognitive problem will appear.

It was the experimental-analytical method for the moments determination, referred to a simple symmetric material body. The accuracy of this method fist of all depends on the accuracy of the component measurements of physical magnitudes. Formulae used for calculations of the mass moments cannot be questioned as they are the definitions of the moments. However, a problem of determination of the mass moments of quite complex solids for which it is impossible to derive univocal exact records of this kind of characteristic magnitudes remains. Thus the experimental method, based on a determined description of the reality, has to be applied. One of the methods is the compound pendulum method, presented among others in $[6,7]$.

Of course, the ring is such a simple solid that the compound pendulum method does not have to be used to determine its mass moments; however, the cognitive aim raised in the paper requires comparison of the former one with the latter experimental-analytical method. The ring is now the compound pendulum with the length $l$ being equal to the internal radius, that is $r$ (Fig. 2).

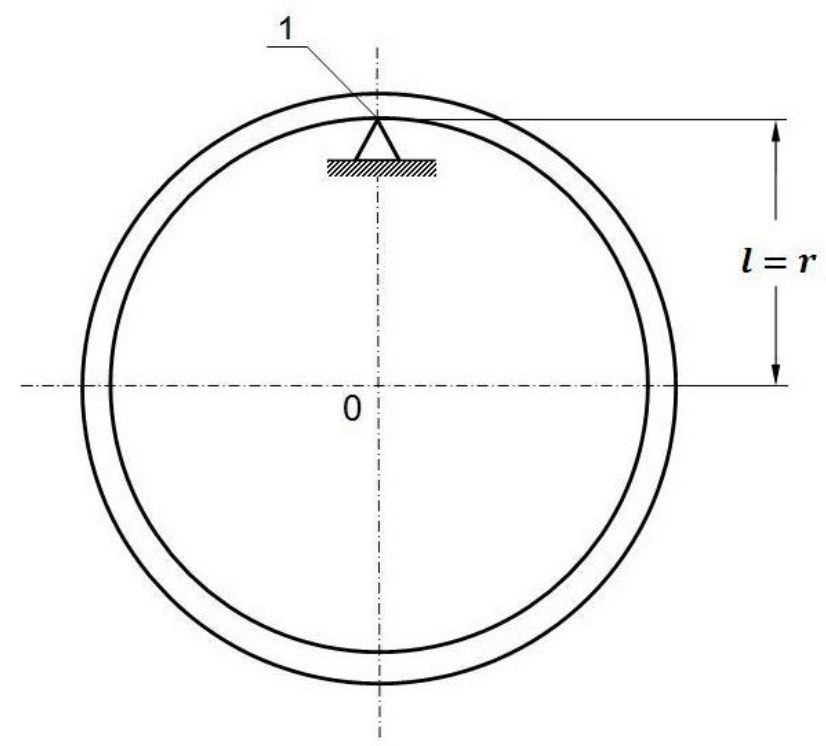

Fig. 2. Ring as the compound pendulum

The theory to the former method is the formula on the mass moment of the compound pendulum, that is the solid suspended (or supported) on the axis being above the centre of gravity. This formula has the following configuration: 


$$
J_{1}^{*}=\frac{m g l T^{2}}{4 \pi^{2}}
$$

where $g$ is the gravitation; $l$ - as mentioned above, is the pendulum length, here equal to the radius $r$; symbol $T$ is the motion period of the compound pendulum. This formula is referred to the mass moment against the axis coming through the ring fulcrum.

The mass $m$, length $l=r$, and period $T$ were measured. The following results were achieved: $m=0.074 \mathrm{~kg} ; r=0.042 \mathrm{~m} ; T=0.54 \mathrm{~s}$. After substituting the gravitation $\mathrm{g}=9.81$ $\mathrm{m} . \mathrm{s}^{-2}$, and measured magnitudes to the formula (4), one obtains

$$
J_{1}^{*}=\frac{0.074 \cdot 9.81 \cdot 0.042(0.54)^{2}}{4 \pi^{2}}=0.225 \cdot 10^{-3} \mathrm{~kg} \cdot \mathrm{m}^{2}
$$

After transformation, the formula (3) is achieved and introducing new notations, one obtains the formula on the mass moment against the axis coming through the centre of gravity, then

$$
J_{0}^{*}=J_{1}^{*}-m r^{2}
$$

that is

$$
J_{0}^{*}=0.225 \cdot 10^{-3}-0.074(0.042)^{2}=0.094 \cdot 10^{-3} \mathrm{~kg} \cdot \mathrm{m}^{2}
$$

There are big differences in the moment values, determined by the method of compound pendulum as the definition formula. Now it is worthy calculating the relative errors - in reference to $J_{0}^{*}$ and $J_{1}^{*}$; they are determined by the following formulae:

$$
\begin{gathered}
\delta_{J_{0}^{*}}=\frac{\left|J_{0}^{*}-J_{0}\right|}{J_{0}^{*}} \cdot 100 \% \\
\delta_{J_{1}^{*}}=\frac{\left|J_{1}^{*}-J_{1}\right|}{J_{1}^{*}} \cdot 100 \%
\end{gathered}
$$

Substituting the values of all the moments, one obtains

$$
\delta_{J_{0}^{*}}=\frac{\left|0.094 \cdot 10^{-3}-0.47 \cdot 10^{-3}\right|}{0.094 \cdot 10^{-3}} \cdot 100 \%=56.4 \%
$$




$$
\delta_{J_{1}^{*}}=\frac{\left|0.225 \cdot 10^{-3}-0.278 \cdot 10^{-3}\right|}{0.225 \cdot 10^{-3}} \cdot 100 \%=23.6 \%
$$

These errors do not result from the inaccuracies of measurements of particular magnitudes; their reason does not lie in the conditions the compound pendulum was swinging. Such big values of the errors may result only from the imperfection of the theory applied with the cause being at the very source. That theory should be substituted by another one, more adequate theory. Further actions are to follow in that direction.

\section{INTO AN ADEQUATE DESCRIPTION OF THE KINETICS OF MATHEMATICAL PENDULUM}

Mathematical pendulum (circular), as reported by references [8-10], is a body, considered as a particle/bob, suspended in a motionless point by a weightless cord/rod from a frictionless pivot, without friction or air resistance. The weightless rod (meaning its mass may be omitted) of the length $l_{0}$ is swinging in a vertical plane under a gravity (Fig. 3). This body/bob, displaced from its resting equilibrium position, is subject to a restoring force due to the gravity and oscillates around the equilibrium position, swinging back and forth [11].

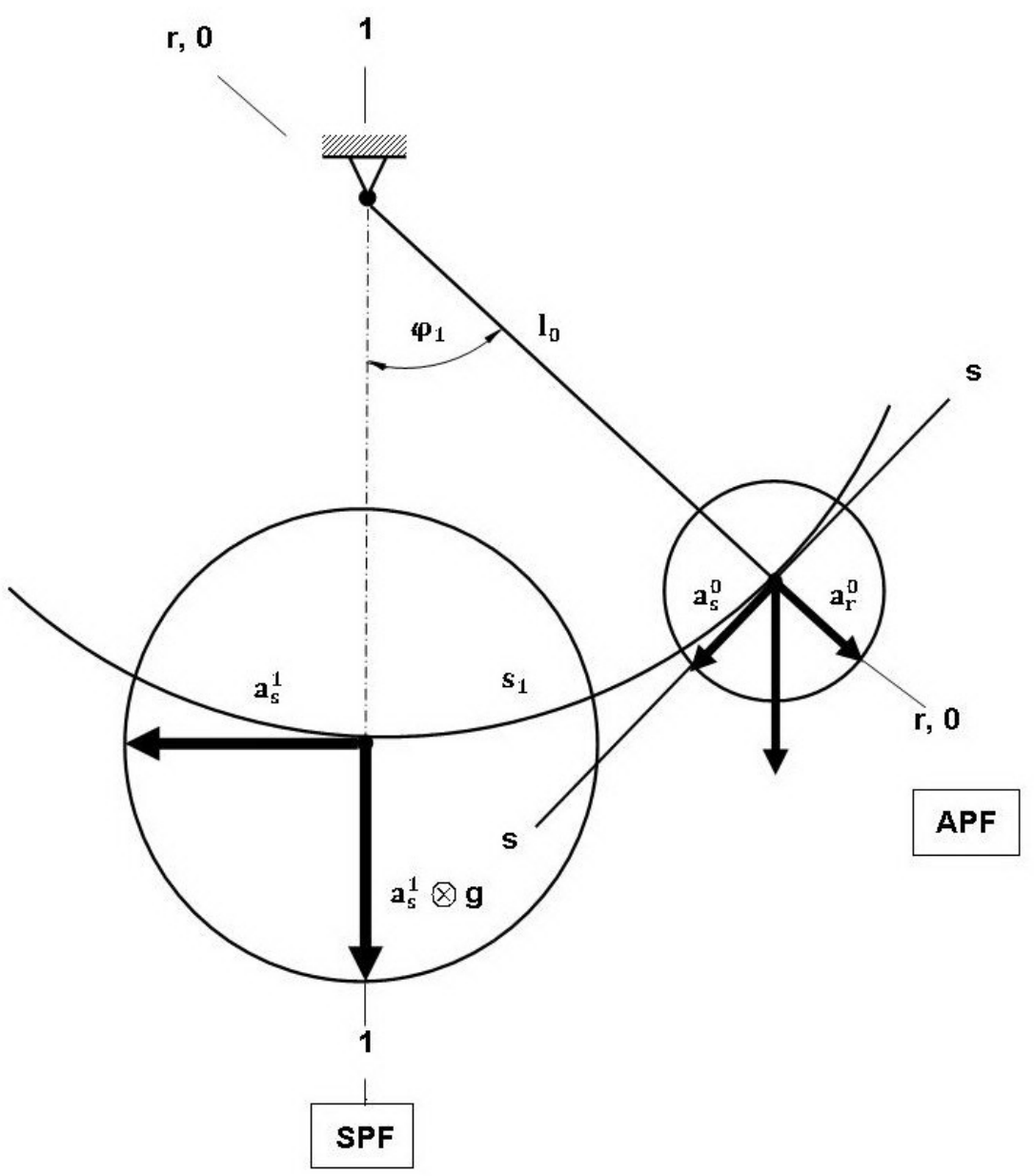

Fig. 3. Scheme of mathematical pendulum and placement of potential fields and accelerations 
Some other references [12-15] treat the description with the same approach, confirming the resulting kinematics of the pendulum. Gravitation influences the pendulum motion but it is not the only reason of the phenomenon. The body suspended on the weightless rod in fact possesses a mass and inertia, therefore also the inertial acceleration (second Newton's law).

The existent theory neglects the acceleration resulting from the body inertia, ignores it and focuses on the gravity force only with the gravitation being its component. There is lack of the second reason of the pendulum swinging which is the inertial acceleration as the component of the acceleration in peripheral direction. Thus the component of peripheral gravitation has been regarded and it is against the nature; for the gravitation acts in radial Earth's direction only (for a local consideration it may be assumed to be perpendicular to the planet surface).

The adequate theory of the mathematical pendulum motion has to take into account these two reasons of the phenomenon, then inertial and gravitational accelerations. Gravitation as a vector does not change any of the four its features: the pivot, direction, sense of a vector, and value. The inertial acceleration changes its value, sense, and direction. Thus the pendulum moves with a variable motion; displaced from its resting equilibrium position, set going it will move first with accelerated variable motion, then with retarded motion, swinging back and forth repeatedly.

A deflected material body (bob as an element of the pendulum) is on an unstable potential field $A P F$ (Fig. 3). Here the gravitation $g$ and inertial acceleration $a$ are present. The inertial acceleration has its radial component $a_{r}^{0}$ and tangent to the bob's trajectory component of the suspended material body, i.e. $a_{s}^{0}$. Symbols $r, s$ denote respective directions: radial $r$ and tangent $s$, whereas zero in the powers meaning zero and terminal bob's positions.

Then the bob moves on angular trajectory $\varphi_{1}$ (linear $s_{1}$ ) to take a stable position, being on the stable potential field $S P F$. At this point the inertial acceleration reaches two times higher value, equal to the gravitation (that relation will be proved further on). The radial component $a_{r}^{1}$ coexists with gravitation, as marked by a special symbol $\otimes$.

There will be only one, first cycle of the pendulum motion considered, as it is necessary to solve the problem. The whole adequate description of kinetics of the mathematical pendulum is to be presented in another paper.

\section{ADEQUATE DESCRIPTION OF ACCELERATED CYCLE OF MATHEMATICAL PENDULUM MOTION}

At the source of cognitive way of any physical phenomena which occur with variable velocity/intensity between the neighbouring energetic states, there is a general differential description, namely

$$
d Z= \pm \frac{\partial Z}{\partial N} d N
$$

where: $d Z$ - total differential of magnitudes being dependent variables, $d N$ - total differential of magnitudes being independent variables, $\frac{\partial Z}{\partial N}$ - partial derivative of magnitudes dependent against independent ones. Symbols $( \pm)$ are the algebraic operators fulfilling a 
determined function. The sign $(+)$ confirms just physical meaning of mathematical description, whereas the sign $(-)$ ascribes the physical meaning to the record.

In solving the differentia equation the initial conditions of any process, and not those connected with the beginning of measurements of determined physical magnitudes, are of importance [16-18]. Measurements of these magnitudes is necessary but not a sufficient condition. The sufficient condition is referred to the measurement at the beginning of a phenomenon. The record of equation (8), applied to the considered phenomenon, has the following configuration:

$$
d s=\frac{\partial s}{\partial t} d t
$$

where the algebraic operator is left positive. That means the length variations of linear path $s$ are rising progressively and the velocity/rate of these variations is rising with the time lapse $t$.

\section{CREATION OF DETAILED CHARACTERISTICS OF PHASE OF ACCELERATED PENDULUM MOTION}

The first detailed characteristics of phase of accelerated pendulum motion is the dependence of the way length on time, that is $s=f(t)$; it refers to exponential, progressively rising curve (Fig. 4).

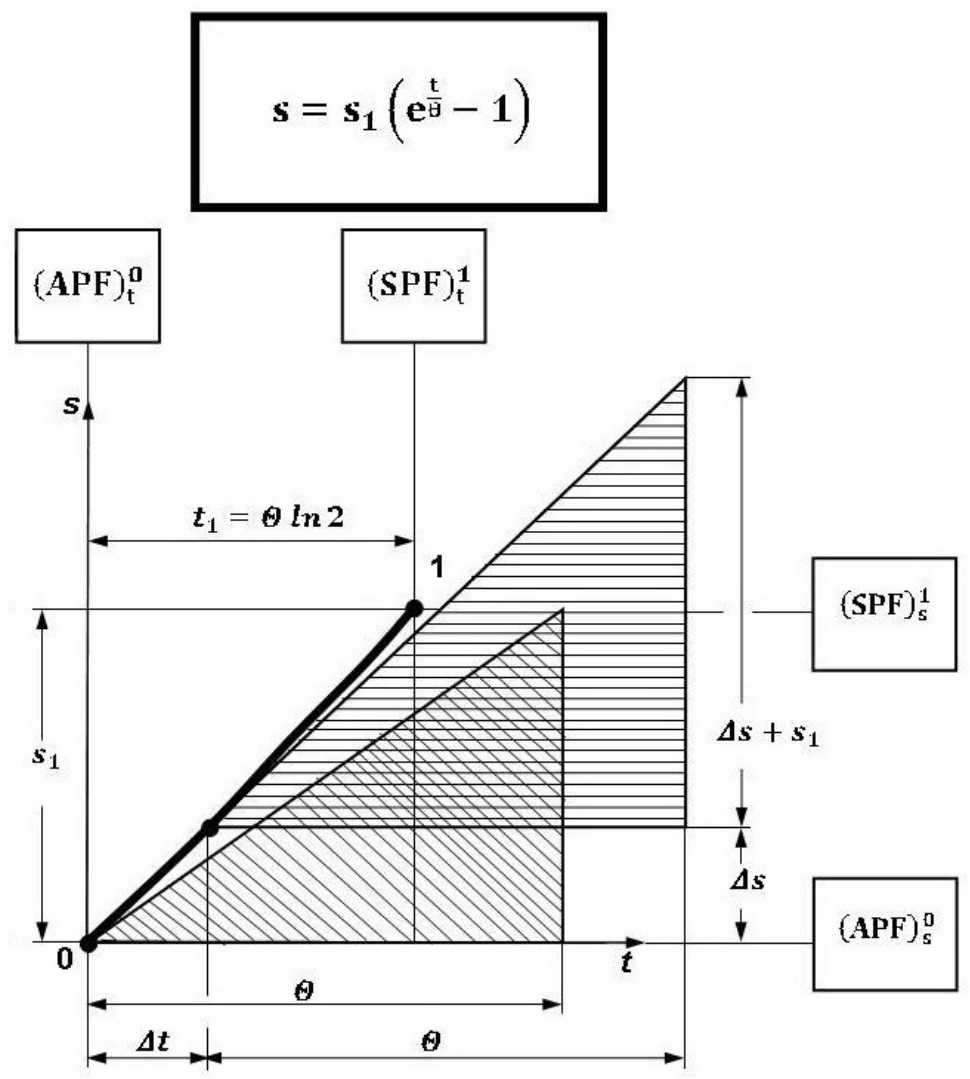

Fig. 4. Illustration of time-path characteristics creation schematic 
This curve is the envelope of right-angled triangles with the time leg being constant and equaled to the so called time constant $\Theta$, and the path leg is changing respectively. The initial point of the curve is on the crossing of unstable potential fields: time unstable potential field $(A P F)_{t}^{0}$ and the path unstable potential field $(A P F)_{s}^{0}$. Terminal point of the curve is situated on the crossing of stable potential fields: time stable potential field $(S P F)_{t}^{1}$ and path stable potential field $(S P F)_{s}^{1}$. The mentioned potential fields are the limits of the time-path space (space-time). The introduced signs $0 ; 1$, being in the powers of the particular potential fields are referred to the corresponding points of the considered indicative curve.

It may be noticed, in the rectangular coordinate system $0, t, s$ it was possible to interpret all the potential fields in more detail; both time and path potential fields have been individuated.

The path leg of this moving right-angled triangle is the property variable which results from the changing position of tangent to the curve of path length. For $\Delta s$ the length of this leg equals $\Delta s+s_{1}$.

Now one may begin integrating the equation (9), denoting limits of the integrals and total differentials. That means

$$
\int_{\Delta s}^{\Delta s+\left(\Delta s+s_{1}\right)} d s=\frac{\partial s}{\partial t} \int_{\Delta t}^{\Delta t+\Theta} d t
$$

and further

$$
\Delta s+s_{1}=\frac{d s}{d t} \Theta
$$

or

$$
\frac{d s}{\Delta s+s_{1}}=\frac{1}{\Theta} d t
$$

One may notice, the partial derivative has been substituted by the quotient of the differentials. It could be done that way because the total differentials have been clearly determined by introducing limits of the integrals.

Furthermore, by integrating both sides of the equation (12), one obtains

$$
\ln \left(\Delta s+s_{1}\right)=\frac{1}{\Theta} t+C^{*}
$$

or

$$
\Delta s+s_{1}=e^{\frac{t}{\Theta}+C^{*}}=e^{C^{*}} \cdot e^{\frac{t}{\Theta}}=C e^{\frac{t}{\Theta}}
$$


After taking into account that for $t=0$ the magnitude $\Delta s=0$, one obtains

$$
C=s_{1}
$$

and after substituting (15) to (14), and regarding that $\Delta s=s$

$$
s=s_{1}\left(e^{\frac{t}{\Theta}}-1\right)
$$

Now one may determine the second coordinate of the point 1 , that is time $t_{1}$. It is gained by introducing the path length $s_{1}$ and the mentioned time to the equation (16). Therefore

$$
t_{1}=\Theta \ln 2
$$

Of course, the equation (16) may be presented in the form to be connected closer with the pendulum characteristics, that is to cover the pendulum length $l_{0}$, deflection angle $\varphi$ and the interstate deflection angle $\varphi_{1}$. Thus one should take into account a connection existing between linear $s_{1}$ and angular path length $\varphi_{1}$, then

$$
s_{1}=\varphi_{1} \cdot l_{0}
$$

so that, after substituting (18) to (16), the following form of dependence is obtained

$$
s=\varphi_{1} \cdot l\left(e^{\frac{t}{\Theta}}-1\right)
$$

Naturally, now further formulae may be created, being derivative magnitudes, that is: velocity $v$, acceleration $a$, and impulse $i$ and further, yet not named magnitudes, respectively. Therefore

$$
\begin{gathered}
v=\frac{d s}{d t}=\frac{\varphi_{1} \cdot l_{0}}{\Theta} e^{\frac{t}{\Theta}}=v_{0} e^{\frac{t}{\Theta}} \\
a=\frac{d^{2} s}{d t^{2}}=\frac{d v}{d t}=\frac{\varphi_{1} \cdot l_{0}}{\Theta^{2}} e^{\frac{t}{\Theta}}=\frac{v_{0}}{\Theta} e^{\frac{t}{\Theta}}=a_{0} e^{\frac{t}{\Theta}}
\end{gathered}
$$




$$
i=\frac{d^{3} s}{d t^{3}}=\frac{d^{2} v}{d t^{2}}=\frac{d a}{d t}=\frac{\varphi_{1} \cdot l_{0}}{\Theta^{3}} e^{\frac{t}{\Theta}}=\frac{v_{0}}{\Theta^{2}} e^{\frac{t}{\Theta}}=\frac{a_{0}}{\Theta} e^{\frac{t}{\Theta}}=i_{0} e^{\frac{t}{\Theta}}
$$

Let us consider the dependence (21), determining the acceleration $a_{s}=a$ for $t=0$. Regarding the time value, one obtains

$$
(a)_{t=0}=a_{0}=\frac{\varphi_{1} \cdot l_{0}}{\Theta^{2}}
$$

For $t_{1}=\Theta \ln 2$ the acceleration $a_{1}$ will be two times higher that results from the formula (21); thus

$$
a_{1}=2 a_{0}=\frac{2 \varphi_{1} \cdot l_{0}}{\Theta^{2}}
$$

Regarding further that $a_{1}=g$, one obtains the formula on the Earth gravitation which may be determined by using the theory of mathematical pendulum. This formula has the following configuration:

$$
g=\frac{2 \varphi_{1} l_{0}}{\Theta^{2}}
$$

and then regarding

$$
\Theta=\frac{t_{1}}{\ln 2}
$$

that results from the formula (17),

$$
g=\frac{2(\ln 2)^{2} \cdot \varphi_{1} \cdot l_{0}}{\left(t_{1}\right)^{2}}
$$

or

$$
g=0.96 \frac{\varphi_{1} \cdot l_{0}}{\left(t_{1}\right)^{2}}
$$


One could determine the pendulum vibration period $T_{0}$, that is the time of its double swing equaling the sum of times $t_{1}$ of four consecutive phases of variable motion, that is $T_{0}=4 t_{1}$. Therefore, regarding $t_{1}$ of the formula (28), the formula on period $T_{0}$ takes the form

$$
T_{0}=3.92 \sqrt{\frac{\varphi_{1} \cdot l_{0}}{g}}
$$

Now, one should determine that Earth acceleration $g$, by using for this purpose the derived formula on this magnitude, or the formula (28). A proper experiment was performed, by measuring the angle of interstate deflection of pendulum of length $l_{0}$, that is the angle $\varphi_{1}$; moreover, the pendulum motion time between the neighbouring states, then $t_{1}$.

Three three-element sets of experimental data $\left(l_{0}, \varphi_{1}, t_{1}\right)$ have been taken into account. They are the following sets: (1) $l_{0}=1 \mathrm{~m}, \varphi_{1}=1 \mathrm{rad}, t_{1}=0.5 \mathrm{~s}$; (2) $l_{0}=0.5 \mathrm{~m}, \varphi_{1}=1 \mathrm{rad}, t_{1}=$ $0.35 \mathrm{~s}$; (3) $l_{0}=0.25 \mathrm{~m}, \varphi_{1}=1 \mathrm{rad}, t_{1}=0.25 \mathrm{~s}$. Substituting these sets of experimental data to the formula (28), the same value of acceleration $g$ equaling $3.85 \mathrm{~m} . \mathrm{s}^{-2}$ was obtained each time. Therefore in the following considerations only this adequate value of the Earth acceleration has been accepted.

\section{DERIVATION OF FORMULA ON THE MASS MOMENT OF COMPOUND PENDULUM}

The formula on the mass moment of a compound pendulum is derived basing on fact that the period of vibration of an elementary mass $m_{0}$, suspended by a cord of length $l_{0}$, being in the axis of the compound pendulum, equals to the vibration period of the former pendulum. Therefore the vibration period of such a situated mathematical pendulum is equal to the compound pendulum vibration period, then $T_{0}=T$.

The inertia moment $M_{B}$ of the physical pendulum equals to the product of its mass moment $J_{1}^{*}$ and the angular acceleration $\varepsilon$, that is

$$
M_{B}=J_{1}^{*} \cdot \varepsilon
$$

and the detailed form of the formula on the inertia moment takes the following configuration

$$
m \cdot a \cdot l=J_{1}^{*} \cdot \varepsilon
$$

The inertia moment of the mathematical pendulum (of elementary mass $m_{0}$, suspended on the cord of length $l_{0}$ ) is described by the dependence

$$
m_{0} \cdot a \cdot l_{0}=m_{0} \cdot l_{0}^{2} \cdot \varepsilon
$$


where $m_{0} \cdot l_{0}^{2}$ is the definition record of the mass moment of this mathematical pendulum.

Equation (32) may be presented in a more simple form, namely

$$
a=l_{0} \cdot \varepsilon
$$

Now, dividing on sides of (31) by (33), that is

$$
\frac{m \cdot a \cdot l}{a}=\frac{J_{1}^{*} \cdot \varepsilon}{l_{0} \cdot \varepsilon}
$$

one obtains

$$
m \cdot l=\frac{J_{1}^{*}}{l_{0}}
$$

so

$$
l_{0}=\frac{J_{1}^{*}}{m \cdot l}
$$

Now, substituting (36) to (29) and regarding that $T_{0}=T$, one obtains

$$
T=3.92 \sqrt{\frac{\varphi_{1} \cdot J_{1}^{*}}{m \cdot g \cdot l}}
$$

Let us transform the formula (37) in view of obtaining the dependence on mass moment of the compound pendulum. Therefore

$$
J_{1}^{*}=\frac{m \cdot g \cdot l \cdot T^{2}}{(3.92)^{2} \cdot \varphi_{1}}
$$

and after regarding that $g=3.85 \mathrm{~m} . \mathrm{s}^{-2}$

$$
J_{1}^{*}=0.25 \frac{\mathrm{m} \cdot \mathrm{l} \cdot \mathrm{T}^{2}}{\varphi_{1}}
$$




\section{VERIFICATION OF THE NEW THEORY}

Let us review and check the theory in practice, i.e. refer it to a compound pendulum, that is the ring of the following characteristics: mass $m=0.074 \mathrm{~kg}$; length $l=0.042 \mathrm{~m}$; its vibration period $T=0.54 \mathrm{~s}$; angle of interstate deviation $\varphi_{1}=47^{\circ}=0.82 \mathrm{rad}$.

Now, let us substitute all these components of the ring characteristics (of the compound pendulum) to the formula (39) on the axis mass moment. Therefore

$$
J_{1}^{*}=0.25 \frac{0.074 \cdot 0.042 \cdot(0.54)^{2}}{0.82}=0.277 \cdot 10^{-3} \mathrm{~kg} \cdot \mathrm{m}^{2}
$$

That mass moment, calculated above acc. to formula (3), equals $0.278 \mathrm{~kg} \cdot \mathrm{m}^{2}$. Therefore the relative error $\delta_{J_{1}^{*}}$ equals

$$
\delta_{J_{1}^{*}}=\frac{\left|J_{1}^{*}-J_{1}\right|}{J_{1}^{*}} \cdot 100 \%=\frac{0.278 \cdot 10^{-3}-0.277 \cdot 10^{-3}}{0.278 \cdot 10^{-3}} \cdot 100 \%=0.36 \%
$$

The ring mass moment, related to the axis coming through its gravity centre, and calculated acc. to formula (5), takes the value

$$
J_{0}^{*}=J_{1}^{*}-m r^{2}=0.277 \cdot 10^{-3}-0.074(0.042)^{2}=0.146 \cdot 10^{-3} \mathrm{~kg} \cdot \mathrm{m}^{2}
$$

That mass moment, calculated above acc. to formula (2), equals $0.147 \cdot 10^{-3} \mathrm{~kg} . \mathrm{m}^{2}$. Therefore in this case the relative error $\delta_{J_{0}^{*}}$ equals

$$
\delta_{J_{0}^{*}}=\frac{\left|J_{0}^{*}-J_{0}\right|}{J_{0}^{*}} \cdot 100 \%=\frac{\left|0.146 \cdot 10^{-3}-0.147 \cdot 10^{-3}\right|}{0.146 \cdot 10^{-3}} \cdot 100 \%=0.68 \%
$$

As may be noticed, the relative errors referred to the mass moments of the studied ring are equal now some hundreds of per cent. It is worthy comparing these errors with the ones obtained using existent grounded theory. The determined error of axial inertia moment of the ring is 83 times higher than the error of this magnitude determined in accordance with the new adequate theory. In relation to the polar inertia moment the coefficient of the correlation equals 66 , so its value is also relatively immense. That proves the existent pendulum theory (both mathematical as well as physical) is completely erroneous; it does not adhere to the studied reality and is not adequate in its character.

A new theory, presented in this paper, is the adequate theory. Very low errors of both these mass moments do not result from its structure and contents, but from an inaccuracy of calculations of both magnitudes and the measurement of the component magnitudes forming determined, used herewith the formulae. 


\section{CONCLUSION}

Each theory should be practical and verifiable. The necessity to determine mass moments of solids is of special importance when characterizing dynamic systems with the solids under variable motion. A precise determination of mass moments of particular elements of a system is possible. The up-to-date existent theory does not allow to do that without introducing different corrective coefficients.

The adequate solution required returning to the source. That allowed to discover unusual thing referring to the value of acceleration which equals $3.85 \mathrm{~m} . \mathrm{s}^{-2}$ [19-22]. The source is the primary description of dependence of pendulum way length on time of its variable motion. As mentioned above, only one phase of variable motion covering accelerated variable motion, has been described. That was enough to derive formulae on mass moments of solids. A complete theory of kinetics of mathematical pendulum (as well as of compound pendulum) will be presented soon.

It is worthy stating that the pendulum motion is quantum in its character. One may note the energetic states, tentative energetic states, where change in the kind of variable motion occur. The accelerated variable motion is transformed into retarded variable motion, this one changes its character again into accelerated one. This variability is characteristic for a pendulum motion. That quantum nature is reflected in some other references [23-28].

Such activities have been just undertaken in reference to a determined fragment of technological macro-reality. The work was performed to describe quantum nature of the tool edge wear as the dependence of tool life under cut on the main velocity [26]. There the analytical description of each interstate fragment of the complex non-continuous curve was performed without any simplification by statistic tools.

\section{References}

[1] K. R. Popper, Logic of Scientific Discovery (in Polish). PWN, Warszawa, 1977.

[2] S. Pabis, Method of empirical sciences - 12 lectures (in Polish). Edit. Office of Politechnika Koszalińska, Koszalin, 2007.

[3] T. Scott, Think like Einstein (in Polish). Edit. Office REBIS, Poznań 2001, 1st Edition (transl. from English).

[4] Zdzisław Pluta, Tadeusz Hryniewicz, Journal of Quantum Information Science (JQIS), 1(3) (2011) 127-134.

[5] M. Mazur, Technical Terminology (in Polish). WNT, Warszawa 1961, 1st Edition.

[6] W. Hajko, Physics in Examples (in Polish). WNT, Warszawa 1967, 1st Edition (transl. from Czech).

[7] T. Drynski, Laboratory Experiments in Physics (in Polish). PWN, Warszawa 1978, 7th Edition.

[8] M. R. Matthews, A. Stinner, C. F. Gauld, The Pendulum: Scientific, Historical, Philosophical and Educational Perspectives, Springer, 2005.

[9] M. R. Matthews, C. F. Gauld, A. Stinner, Science \& Education, 13 (2005) 261-277.

[10] S. Ziemba, Vibration Analysis (in Polish). PWN, Warszawa 1957. 
[11] R. Nelson, M. G. Olsson, American Journal of Physics, 54(2) (1986) 112-121. DOI: $10.1119 / 1.14703$.

[12] K. Piszczak, J. Walczak, Vibrations in Machine Building (in Polish). PWN, WarszawaKraków, 1967, 2nd Edition.

[13] Z. Osinski, Theory of Vibrations (in Polish). PWN, Warszawa, 1978, 1st Edition.

[14] R. Hooke, D. Shaffer, Mathematical Models and the Reality (in Polish). PWN, Warszawa, 1969 (transl. from English).

[15] J. R. Taylor, Classical Mechanics, Vol. 1 (in Polish), Wydawnictwo Naukowe PWN, Warszawa, 2006 (transl. from English), 1st Edition.

[16] Zdzisław Pluta, Fundamentals of Smoothing Using Elastic Abrasive Wheels (in Polish) Edit. Office of Politechnika Koszalińska, Koszalin, 2007.

[17] Zdzisław Pluta, Tadeusz Hryniewicz, Intern. Letters of Chemistry, Physics and Astronomy 1 (2013) 1-16.

[18] Zdzisław Pluta, Tadeusz Hryniewicz, Intern. Letters of Chemistry, Physics and Astronomy 1 (2013) 17-32.

[19] Zdzisław Pluta, Tadeusz Hryniewicz, Intern. Letters of Chemistry, Physics and Astronomy 2 (2012) 28-34.

[20] Zdzisław Pluta, Tadeusz Hryniewicz, Intern. Letters of Chemistry, Physics and Astronomy 3 (2012) 1-10.

[21] Zdzisław Pluta, Tadeusz Hryniewicz, Intern. Letters of Chemistry, Physics and Astronomy 3 (2012) 11-23.

[22] Zdzisław Pluta, Tadeusz Hryniewicz, Intern. Letters of Chemistry, Physics and Astronomy 4 (2012) 1-7.

[23] J. Magueijo, Faster than Light (in Polish). Edited by Amber, Warszawa, 2003 (transl. from English), 1st Edition.

[24] P. Atkins, Galileo Finger (in Polish). Edit. Office REBIS, Poznań, 2006 (transl. from English), 1st Edition.

[25] M. Chown, Quantum Theory Does not Bite. Guide through the Universe (in Polish). Wydawnictwo Zysk and Co., Poznań, 2009 (transl. from English), 1st Edition.

[26] Zdzisław Pluta, Tadeusz Hryniewicz, Intern. J. Adv. Manuf. Technol. 51 (2010) 35-43. DOI: $10.1007 / \mathrm{s} 00170-010-2595-5$.

[27] Zdzisław Pluta, Tadeusz Hryniewicz, Intern. J. Adv. Manuf. Technol. 62 (2012) 529- 542. DOI: 10.1007/s00170-011-3813-5.

[28] Zdzisław Pluta, Tadeusz Hryniewicz, J. Modern Physics. 3 (2012) 625-635. DOI: 10.4236/jmp.2012.37086. 